\title{
SEMÁNTICA INTERPRETATIVA Y TEORÍA SEMIÓTICA
}

\author{
Teresa Espar
}

(Universidad de Los Andes, Venezuela)

\section{INTERPRETACIÓN}

La semántica interpretativa de Katz, Fodor y Postal (1983) no es una teoría semántica. La llamada semántica interpretativa de François Rastier (1987), se presenta como una teoría semántica. Un cuarto de siglo separa una proposición de la otra sin que nos sea permitido justificar esta homofonía partiendo de posiciones comunes de carácter epistemológico, teórico o metodológico. Precisamente la lingüística de esta época nos tiene habituados a este paradójico extravío, porque una cosa es semántica interpretativa y otra, muy diferente, semántica interpretativa, como señalábamos al principio.

Interpretación no es, no dice lo mismo; un mismo sonido no es un sentido único; hay una expresión para varios contenidos o una forma con otra función comunicativa. El concepto de interpretación es utilizado en semiótica en dos sentidos muy diferentes que dependen de los postulados 
de base a los que se refiere ${ }^{1}$ y sobre todo de la idea que se tenga de la forma semiótica. Posner (1978: 71) la define como una actividad que hace explícito lo que está implícito en un texto dado.

La semántica generativa sigue la concepción clásica propia de las escuelas polaca y vienesa de lógica. Esta concepción opone la forma al contenido (o fondo) y postula que todo sistema de signos puede ser descrito de manera formal haciendo abstracción de su contenido: de este modo una lengua natural podrá ser descrita como un sistema de expresión - siguiendo la terminología hjlemsleviana - susceptible de recibir posteriormente una interpretación semántica. En semántica generativa ésta tendrá como finalidad elaborar reglas de carácter formal que asignen una interpretación semántica a las estructuras profundas que son de carácter sintáctico, es decir, desprovistas de significación. Estas reglas no pueden sino reposar en los conceptos epistemológicos de gramaticalidad y aceptabilidad, que nada aportan a la resolución de los problemas de una teoría semántica.

Para la tradición saussuriana, sobre todo para aquella lingüística cuyos fundamentos epistemológicos provienen de la fenomenología de Husserl, un signo debe ser definido por su significación entendiendo la forma semiótica como significancia. Interpretar no será, entonces, atribuirle un contenido a una forma, sino producir una paráfrasis, traducir una unidad significante de un sistema semiótico en otro; esta concepción parte de la postura que defiende la correlación entre los planos de la expresión y del contenido.

Luis Hjlemslev entiende por interpretación el hecho de atribuirle un uso a una forma semiótica en el sentido en el que se puede entender en las semióticas estéticas; así interpretar será reproducir o ejecutar (interpretación de una obra de teatro o de una obra musical, por ej.). De ahí que este autor pueda afirmar que ningún sistema semiótico es, en principio, interpretado y que todos son interpretables.

Cuando de lo que se trata es de producir un modelo teórico de la competencia del enunciatario, la interpretación tomará el sentido de lectura. La teoría semiótica describe la producción y la interpretación del sentido y nos proporciona, por un lado, un modelo de la competencia del enunciador al describir el recorrido generativo de la producción del sentido; por otro lado, un modelo de la competencia del enunciatario que debería ser, naturalmente, intepretativa. Este componente interpretativo de la teoría semiótica dará cuenta de la re-producción del sentido en la lectura, entendida como puesta en correlación de los planos de la expresión y del contenido. Esta perspectiva diferencia también la gramática generativa y la semiótica europea. Para la gramática generativa las transformaciones que desembocan en la manifestación de las formas de base en las estructuras de superficie no

1 Véase Greimas-Courtés (78 y 86: s.v. «Interpretation», «Herméneutique», «Lecture»). 
conllevan modificaciones de contenido, mientras que para la semiótica, el recorrido generativo de la significación que supone el paso de las estructuras profundas a las de superficie, implica una suerte de ganancia, de aumento en el revestimiento semántico al pasar de una instancia $a b$ quo, más abstracta, a una instancia ad quem mucho más rica en sus componentes; esta re-construcción de los niveles de articulación es su forma interpretativa.

En sentido corriente, la interpretación está también intimamente ligada a la hermenéutica. La hermenéutica no es sino una disciplina interpretativa, referida esencialmente a los textos filosóficos y religiosos, que pone en juego las relaciones de éstos con el referente, con las condiciones extralingüísticas de la producción y lectura de esos textos. Hace intervenir el contexto socio-histórico y trata de desentrañar todas las interpretaciones aceptables de un discurso; sus criterios evaluativos incluyen una posición filosófica de referencia. A la interpretación hermenéutica no se le reconoce status científico ya que se propone realizar una suerte de lectura singular de cada texto original, haciendo intervenir las nociones de referencia y de sujeto discursivo. El sentido sería, además, inmanente al texto; la interpretación, fundada en la revelación, desvelaría lo que previamente habría sido depositado en él por Dios o por los hombres.

La hermenéutica contemporánea amplía sus territorios y nos remite a unos modos de interpretación de textos literarios o de otros textos que permiten llamarla hermenéutica profana y de vanguardia (Rastier 1989: 21 ss.) y que incluye al psicoanálisis neo-freudiano inspirado en el pensamiento de Lacan. Esta tendencia ha dominado los debates sobre la interpretación de textos literarios debido al extraordinario éxito de intérpretes míticos, entre los cuales citaremos como representante arquetípico a Roland Barthes (1973) ${ }^{2}$. Lo más curioso es que esta hermenéutica pulsional se apoya en dos autores ilustres; por un lado, en el Saussure nocturno de los Anagramas y los versos saturnianos y, por otro lado, en Freud. J. Derrida con su teoría de la diseminación y Lacan con su teoría del significante son los líderes intelectuales, creadores de escuela, que responden a esta influencia y que hablan en parábolas.

Esta corriente, de lleno arraigada en la cultura occidental, nos acostumbró y nos deslumbró también con trabajos pioneros como los de Fonagy, Jakobson y Kristeva sobre el valor simbólico, por ej., de los fonemas y los sonidos. En versión lacaniana llamada integrista por Rastier (cf. supra), llega a ignorar las estructuras fundamentales de la textualidad particularmente en su nivel semántico, apoyando su estrategia interpretativa en la negación o mejor en el alejamiento del sentido del texto. El sentido y la significación se ignoran en nombre de la supremacía del significante.

${ }^{2}$ En este libro se encuentran los fundamentos de la hermenéutica de vanguardia que tanta influencia ejerció y ejerce todavía. 
La manera de interpretar - las frases, los discursos, los textos- dependerá sobre todo del tipo de objetividad que le reconozcamos a un texto dado o, dicho en términos tradicionales y ya bien consolidados, el punto de vista construirá el objeto.

La Interpretación en el sentido de la semántica (Katz, Fodor, Postal), de la semántica estructural europea (Hjlemslev), de la hermenéutica clásica (Scheleimacher y Dilthey), hermenéutica moderna (Gadamer y Ricoeur) o vanguardista (Lacan, Derrida, Kristeva), en fin, de la semiótica francesa (Greimas), es, como vimos, uno de los conceptos dificiles y al mismo tiempo centrales para todos aquellos que se ocupan de semántica, entendida ésta como una propuesta interpretativa capaz de generar los modelos de desentrañamiento de la significación.

El interés de esta reflexión que parte de la polisemia del concepto de interpretación sirve para mostrar, aunque sea oblicuamente y antes de adentrarnos en la discusión sobre una teoría semántica, los numerosos puntos de encuentro entre algunas disciplinas con la ciencia que nos ocupa, observándola únicamente desde el punto de vista elegido, es decir, la semántica en su versión interpretativa y la interpretación como objetivo de acercamiento a los textos y a la significación que conllevan.

\section{TEORÍA SEMÁNTICA}

¿Es o no es teoría semántica la llamada semántica interpretativa? Veíamos que en su versión generativa no constituye algo aparte de la teoría sintáctica que la sustenta. La interpretación semántica se limita a proporcionar una serie de reglas de carácter formal que asignan una complementación de componente semántico a la estructura sintáctica de la oración; ni en la época de las proposiciones iniciales de Katz y Fodor, posteriormente de Katz y Postal, ni en otras confrontaciones a las que está habituada la lingüística americana podemos decir que la semántica generativa proponga una teoría semántica ${ }^{3}$ en el sentido en el que entenderemos teoría en este texto. Nos interesa por lo tanto discutir, a partir de este momento, esa propuesta del lingüista y semiótico francés, F. Rastier, todavía poco difun-

${ }^{3}$ Véase al respecto el excelente trabajo de Galmiche (1975), que concluye, después de haber sometido a exposición y discusión más de veinte años de debates en semántica generativa, que ésta no constituye una nueva teoria lingüística transformacional, entre otras razones ya dadas, por entrañar únicamente dicha interpretación un análisis de la actuación del oyente. Lo cierto es que la semántica generativa, a pesar de haber estimulado desde el principio una cierta reflexión sobre los problemas de la significación, debido a su anti-semantismo de principio y a su insuficiente preparación para el debate teórico en este aspecto, no ha logrado impresionar ni a lingüistas semánticos, ni a lógicos, ni a pragmáticos; menos aún, quizás, a los filósofos del lenguaje. 
dida y que se presenta como semántica interpretativa y se define como una teoria semántica, componencial, diferencial y dinámica, Rastier (1989: 8).

Rastier (1987) parte de unas preguntas muy sencillas: ¿Qué se hace cuando se lee un texto?; ¿de dónde procede el sentimiento de su unidad? Se supone que el lector opera a partir de unidades semánticas y poder definir y describir esas unidades en el interior de una teoría, podrá contribuir a dar respuesta a esas preguntas.

Esa teoria podrá confundirse con los acercamientos de la psicología cognitiva que se interesa por las operaciones que realiza un lector para interpretar un texto no coincide con esas operaciones aun cuando las pueda derterminar y también limitar. La teoría interpretativa que discutimos es de clara genealogía lingüística europea y diverge de la semántica cognitiva de inspiración americana.

La semántica cognitiva, como muy bien dice Kleiber (1990: 9) «à le vent en poupe» en este momento entre lingüistas y psicolingüistas sobre todo, pero su aporte está limitado a la semántica léxica y está más cerca de la psicología cognitiva que de la lingüística ${ }^{4}$. La proposición de Rastier intenta delimitar previamente los confines y propósitos de esta búsqueda estableciendo como uno de los objetivos de la investigación, el dar cuenta de la cohesión textual. Aunque otras disciplinas cercanas - la semiótica, la poética, la estilística e incluso los teóricos de la inteligencia artificial- se preocupan también por la cohesión de los textos, se considera que la disciplina que debe ocuparse de ello es en primer lugar la lingüística y dentro de ella, particularmente la semántica: «Sans postuler une linguistique textuelle autonome, il s'agit de décrire le texte comme une région de l'objet linguistique, en précisant sa spécificité et ses relations avec les paliers de l'énoncé et du comme une region de l'objet linguistique, en précisant sa spécificité et ses relations avec les paliers de l'énoncé et du morphème. Pour rendre compte de la cohesion textuelle, il faut élaborer des concepts comme celui d'isotopie, qui ne soient pas directement dépendants des structures syntaxiques et restent donc indiférents a la pretendue limite de la phrase», Rastier (1987: 9-10).

Una nueva opción sitúa esta teoría claramente en el ámbito de las ya bien conocidas y establecidas semánticas del discurso; de evidente filiación greimasiana, el concepto de isotopía ${ }^{5}$, que desde los inicios disfrutó de

\footnotetext{
${ }^{4}$ Puede consultarse su reciente libro La sémantique du prototype en el que expone, apoya y discute las propuestas de Eleanor Rosch, de Berkeley. Con gran provecho, por la precisión de la discusión que emprende al respecto, conviene leer el reciente trabajo de Eugenio Coseriu sobre "Semántica estructural y Semántica Cognitiva» (1990), de particular interés también por el punto de vista que transmite sobre la semántica léxica europea y americana.

${ }^{5}$ Recordemos que uno de los metatérminos más felices y celebrados de la semiótica greimasiana es precisamente el de isotopía. La isotopía, las isotopías, son el hilo teórico conductor de las proposiciones de Rastier (ver Greimas-Courtés: 1978, 1986); una extensa bibliografia previa sobre isotopía se encontrará en los trabajos del autor mencionado, sobre todo en Rastier (1987).
} 
muy buena prensa y se mostró como un enfoque de gran eficacia analítica, proporciona precisamente la posibilidad de la integración de niveles en la reconstrucción del recorrido generativo de la significación. Por otro lado, el hecho de que en el concepto de isotopía esté implícita la idea de cohesión sintagmática de las unidades semánticas, supone que toda proposición de análisis traspasará necesariamente los estrechos límites de la frase.

Pretende también alejarse del inmanentismo del texto practicado por un estructuralismo riguroso y cauteloso al mismo tiempo. Los enfoques inmanentistas que le han supuesto, a las teorías y prácticas lingüísticas de nuestro siglo, una mirada hacia dentro rica en hallazgos, comienza ya a mostrarse como limitada ya que las diversas proposiciones venidas de la pragmática y de las teorías sobre el discurso y la enunciación permiten un enriquecimiento permanente del debate, del objeto y del punto de vista. No olvidemos además que las teorías del discurso abrieron nuevas vías al estudio de efectos de sentido, hasta ahora confinados, como los pasionales.

Desde esta perspectiva, un texto no contiene todo lo que conlleva su interpretación; una vuelta a la filología podría ser necesaria, sin olvidar las imprescindibles relaciones de la semántica interpretativa con la filosofia del lenguaje, cuyos aportes a través de la semántica formal, las cuestiones del referente o de la verdad, la intencionalidad de los actos de habla o las teorías de la interacción conversacional ${ }^{6}$, contribuyen a producir esa apasionante discusión que envuelve hoy a las ciencias del lenguaje. Todo el cuidado para elaborar una teoría semántica estará en la construcción de su coherencia interna, en no perderse de nuevo en el subjetivismo, el contextualismo o un inmanentismo esterilizante: «Évitant de choisir entre des théories non contextuelles du sens, et des théories non linguistiques du contexte, nous avons cherché à intégrer à une sémantique rationnelle de l'interprétation les facteurs que l'on dit trop commodement pragmatiques» (Rastier, 1987: 11).

Rastier parte de la concepción de la isotopía para formular una teoría semántica interpretativa. Una isotopía se establece gracias a una serie de relaciones de identidad entre semas que inducen relaciones de equivalencia entre sememas. El problema que se plantea es que ese módulo de equivalencias no está dado de antemano y hay que acudir a la inferencia para poder identificarlo. El lector provisto en cada caso de una competencia interpretativa diferente, que dependerá de sus conocimientos enciclopédi$\cos$, escogerá de acuerdo con esa competencia una estrategia adecuada y naturalmente condicionada, que proporcionará descripciones diferentes del texto, aun estando provisto de una metodología idéntica. De este modo y a partir de esta experiencia descubrirá el reduccionismo de ciertas prácticas

${ }^{6}$ Ver, entre otros, los trabajos de Montague: Formal Philosophy, de Quine: Word and object, y los de la microsociología de Goffmann. 
analíticas del estructuralismo que proponen una lectura única. Un comentario aparte merece también otra opinión de Rastier que postula un procedimiento llamado por él paradójico, que conduce del texto a los elementos; para ello es necesario partir de la presentación de la isotopía que permite actualizar los semas: «on voit ici une application élémentaire d'un principe général: tout sens, même au palier micro-sémantique, est le produit d'opérations d'interprétation et demeure relatif à une stratégie» (Rastier, 1987: 12).

El nivel micro-semántico es el nivel de análisis sémico o componencial, conocido también como análisis en rasgos distintivos que constituye toda una época (años 60 a 70) del análisis semántico; esa semántica analítica o estructural léxica en su vertiente europea, tiene su correspondiente en América, por la misma época, con una semántica llamada componencial, cuyos límites los impone la frase y que hemos comentado anteriormente como semántica interpretativa americana. El concepto de isotopía es precisamente uno de los que establecen la diferencia entre esa micro-semántica, subsidiaria de un estructuralismo universalista, atomista y combinatorio, seguidor de Leibniz y de su proyecto de una lengua característica, para llegar a este otro estructuralismo, gestaltista - no universalista-, en el que la clase determina al elemento, lo global a lo local y concibe la unidad semántica como elemento de un conjunto significante que no puede ser aprehendido en forma aislada, como unidad de inventario léxico sino como conjunto enunciado.

Para alejarse de esa micro-semántica combinatoria, los semas no podrán ser considerados ni como universales, ni como cualidades del referente, ni como partes de conceptos; no serán además poco numerosos ni unidades últimas o mínimas de un análisis componencial ${ }^{7}$. Entendidos como elementos de un semema entran de este modo a formar parte de una isotopía que los integra al enunciado; forman parte de la cohesión textual, distanciando así de nuevo esta teoría de la que más arriba llamábamos semántica analítica.

${ }^{7}$ La definición que Rastier (1987) da de sema en Glossaire es funcional en el sentido hjlemsleviano del término: «élément d'un sémème, défini comme l'extremité d'une relation fonctionnelle binaire entre sémèmes». Es un funtivo que contrae función con una unidad más extensa; semema es definido como "contenido de un morfema» y morfema como signo mínimo; no hay naturalmente, definición de "signo mínimo". El esfuerzo de formalización en estas definiciones no beneficia mucho al estudioso o al especialista, habituado a manejar una concepción bastante cómoda de sema, aunque no ortodoxa para una teoria de inspiración hjlemsleviana. Nótese que «funcional» en el sintagma «relation fonctionnelle» no es hjlemsleviano sino praguense -si acordamos reconocerle al C.L.P. el mérito de haber propuesto la equivalencia entre función- comunicación o uso. El sema tiene valor por su uso funcional en el discurso, en el enunciado, que es donde contrae función con el semema, unidad, aunque no se diga, más extensa que el sema. 


\section{3. ¿UNA NUEVA TEORÍA?}

Para construir esa teoría semántica capaz de definir las unidades semánticas y de describir sistemáticamente sus relaciones, será necesario definir cuidadosamente los límites impuestos a esta perspectiva y que podríamos resumir, siempre leyendo a Rastier, como:

a) diferenciación en relación con la psicología cognitiva,

b) afirmación inicial de una perspectiva discursiva que busca, como otras teorías, dar cuenta de la cohesión textual,

c) alejamiento del inmanentismo,

d) la semántica se encuentra a medio camino entre la lingüística y la filosofía del lenguaje.

Este conjunto de opciones teóricas, se continúa en la exposición de la incidencia que esas opciones tienen en la concepción de las isotopías que permiten al autor embarcarse en una minuciosa exposición de su teoría interpretativa, completamente estructurada alrededor del concepto central de isotopia, que se desarrolla como una suerte de constelación clasificatoria, paralizadora por lo casuística, pero algo deslumbrante por la finísima sensibilidad categorizadora que demuestra. No obstante, algo, un extraño efecto de sentido, recuerda las agotadoras construcciones de la escolástica decadente. De isotopía en isotopía, de tipo de sema, en sema, de nivel en nivel, el constructo teórico, la metodología de análisis propuesta, se nos presenta como poco eficaz. El afán por alejarse del esquematismo de los modelos universalistas, distanciados de lenguas naturales concretas (las gramáticas universales de Montague, Chomsky y Shaumyan), de su cultura y de su historia, tiene serias consecuencias teóricas en estas proposiciones.

Reconstruir, de este modo, de nivel en nivel los componentes semánticos, previamente definidos como semas aferentes, específicos o genéricos, etcétera., hasta lograr una matizada y compleja descripción de esa conjunción de micro-componentes que estructuran la significación, produce ganancias en la inteligibilidad del texto. Éste es uno de los objetivos de la ciencia, que ya no se plantea en la episteme de nuestra época desentrañar la realidad, decir la verdad sobre el mundo o sobre el lenguaje que lo representa. Este objetivo puede ser logrado a partir de la semántica interpretativa expuesta. Pero las cuestiones del principio: «¿qué se hace cuando se lee un texto? o ¿de dónde procede el sentimiento de su unidad?» requerirían, si quieren que la respuesta concuerde con las exigencias de las ciencias sociales y humanas, precisamente, de algo que esta teoría -en todo caso débil- no proporciona: los modelos de la competencia interpretativa del enunciatario. 
En efecto, la reconstrucción de las isotopías, la puesta en evidencia de la articulación micro-semántica de la significación, podrá explicar la cohesión textual y responder de un modo muy de la prosa de Monsieur Jourdan a las preguntas que se formulaban previamente. Es así como el sentimiento de la unidad de un texto procede de su cohesión y la cohesión de la construcción isotópica en haces, líneas, redes y constelaciones sémicas de las microscópicas unidades semánticas, que, por fin, se manifiestan linealmente, pero no son modelos de la competencia. Los numerosos gráficos no logran ocultar las insuficiencias de formalización, de producción de modelos abstractos de esta teoría.

Los procesos cognoscitivos de abstracción, capaces de inferir a partir de lo particular y de lo empírico, las condiciones generales - universales también- de producción y aprehensión del sentido, son formalizables y entonces «representables» por medio de modelos. Estos modelos concebidos como formaciones descriptivas obtenidas después de propuestas hipotéticas y deductivas son constructos apoyados en el nivel metalingüístico y no deben confundirse con ciertas formas de ordenamientos gráficos de los componentes del enunciado, que de alguna manera tratarían de organizar, representar o transponer unas formas de la expresión en otras. No se trata de «dibujar» los modos de articulación semántica del texto para alejarse así de lo que es imprescindible que haga una teoría: sacrificar lo particular a lo universal, lo concreto a lo abstracto, el uso al esquema.

Los instrumentos que propone Rastier podrán constituir un buen método interpretativo de textos, pero saber lo que dice o quiere decir un texto, no proporciona un modelo de la competencia interpretativa. La ganancia es pérdida desde el punto de vista teórico y el constructo formal esperado es tan sólo un dibujo. Rastier, en sus dos obras del 87 y del 89 pasa revista, en una admirable demostración de cultura enciclopédica sobre teorías semánticas y semióticas, lingüísticas y filosóficas, a la casi totalidad de proposiciones, metodologías y acercamientos diversos a los textos. La erudición y el talento no exentos de bizantinismo, ponen de manifiesto, sin embargo, un problema mayor: la falta de constructos teóricos. La ausencia de modelos formales de la competencia interpretativa coloca esta proposición entre las teorías llamadas débiles; ni coherente, ni exhaustiva ni simple, a la manera de Hjlemslev seguido fielmente por Greimas, al que, no obstante invoca a menudo. Greimas, como sabemos, propone una teoría semántica generativa, sintagmática y general (Greimas-Courtés, 1978: s.v. Sémantique), concebida además y sobre todo como un metalenguaje, como constructo diferenciado - otro discurso, el metadiscurso científico- capaz de producir un modelo explicativo, generativo, interpretativo, adecuado e intelegible diferenciado, de su objeto de estudio, en este caso, todo conjunto significante y no una lengua natural.

La semántica interpretativa de François Rastier, su teoría del texto, aparece confusa, barroca, demasiado casuística. Incapaz de soportar las acu- 
saciones que formula contra el universalismo de principio, la involución especulativa o el formalismo, se aleja así del modelo interpretativo que pretende proporcionar y que se manifiesta como necesario a toda ciencia, para caer en la ineficacia y dispersión interpretativas de las categorizaciones multiplicadas.

No obstante, su competencia de lector agudo, la finura metodológica de que hace gala y la firmeza con la que se aboca a la demostración de que el objeto de la semántica es interpretar, describir, explicitar y mostrar la cohesión y la coherencia de los textos, hacen que sus trabajos más recientes sean imprescindibles en la cultura semántica y semiótica de nuestra época.

\section{Referencias Bibliográficas}

BARTHES, R. (1973). Le plaisir du texte. Paris: Du Seuil.

COSERIU, E. (1962). Teoria del lenguaje y lingüistica general. Madrid: Gredos.

- (1962). «Sistema, norma et parola». En Studi Linguistici in onore di Victtore Pisani, 235-253. Brescia: Paideia Editrice.

- (1976). «L'étude fonctionnelle du vocabulaire. Précis de léxematique»: Cahiers de lexicologie 29, 5-23.

- (1990). «Semántica estructural y semántica cognitiva». En Homenaje al Prof. Francisco Marsá, 239-282, Barcelona: Ed. Universitat de Barcelona.

DUCROT, O. TODOROV, T. (1972). Dictionnaire encyclopédique des sciences du langage. Paris: Seuil.

FERnÁNDEZ, A. R. y otros (1984). Introducción a la semántica. Madrid: Cátedra.

Galmiche, M. (1980). Semántica generativa. Madrid: Gredos.

GreIMAS, A. J. (1986a). Sémantique structurale. Paris: Larousse.

— et COURTES, J. (1979 y 1986). Sémiotique. Dictionnaire raisonnée de la théorie du Langage (I et II). Paris: Hachette.

- et RASTIER, F. (1968). "The interaction of semiotics constraints». Yale French Studies 41, 86-105.

- et Fontanille, J. (1991). Sémiotique des Passions. Paris: Seuil.

HJLEMSLEV, L. (1968-1971). Prolégoménes à une théorie du langage. Paris: Minuit.

KLeIBER, G. (1990). La Sémantique du prototype. Catégories et sens lexical. Paris: P.U.F.

POSNER, R. (1978). «Linguistic tools and literary interprétation». P.T.L. 3, 71-93.

RASTIER, F. (1972). «Systématique des isotopies». En Essais de Sémiótique poétique Greimas, A.J. (ed.). Paris: Larousse.

- (1973). Essais de sémiotique discursive. Tours: Mame.

- (1987). Sémantique interprétative. Paris: P.U.F.

- (1989). Sens et contexte. Paris: Hachette. 